\title{
Generation of Gravity Waves by Pedal-Wavemakers
}

\author{
Isis Vivanco ${ }^{1}$, Bruce Cartwright ${ }^{2,3}$, A. Ledesma Araujo ${ }^{1}$, , Leonardo Gordillo ${ }^{1}\left(\mathbb{D}\right.$ and Juan F. Marin ${ }^{1, *(\mathbb{D})}$ \\ 1 Departamento de Física, Facultad de Ciencia, Universidad de Santiago de Chile, Usach, Av. Ecuador 3493, \\ Estación Central, Santiago 9160000, Chile; isis.vivanco@usach.cl (I.V.); aledesma@pucp.edu.pe (A.L.A.); \\ leonardo.gordillo@usach.cl (L.G.) \\ 2 Pacific Engineering Systems International, 277-279 Broadway, Glebe, NSW 2037, Australia; \\ brucec@esi.com.au \\ 3 School of Engineering, The University of Newcastle, Callaghan, NSW 2308, Australia \\ * Correspondence: juan.marin.m@usach.cl
}

Citation: Vivanco, I.; Cartwright, B.; Ledesma Araujo, A.; Gordillo, L.; Marin, J.F. Generation of Gravity Waves by Pedal-Wavemakers. Fluids 2021, 6, 222. https://doi.org/ $10.3390 /$ fluids 6060222

Academic Editors: Richard Manasseh and Alberto Alberello

Received: 30 April 2021

Accepted: 10 June 2021

Published: 13 June 2021

Publisher's Note: MDPI stays neutral with regard to jurisdictional claims in published maps and institutional affiliations.

Copyright: (c) 2021 by the authors. Licensee MDPI, Basel, Switzerland. This article is an open access article distributed under the terms and conditions of the Creative Commons Attribution (CC BY) license (https:// creativecommons.org/licenses/by/ $4.0 /)$.

\begin{abstract}
Experimental wave generation in channels is usually achieved through wavemakers (moving paddles) acting on the surface of the water. Although practical for engineering purposes, wavemakers have issues: they perform poorly in the generation of long waves and create evanescent waves in their vicinity. In this article, we introduce a framework for wave generation through the action of an underwater multipoint mechanism: the pedal-wavemaking method. Our multipoint action makes each point of the bottom move with a prescribed pedalling-like motion. We analyse the linear response of waves in a uniform channel in terms of the wavelength of the bottom action. The framework naturally solves the problem of the performance for long waves and replaces evanescent waves by a thin boundary layer at the bottom of the channel. We also show that proper synchronisation of the orbital motion on the bottom can produce waves that mimic deep water waves. This last feature has been proved to be useful to study fluid-structure interaction in simulations based on smoothed particle hydrodynamics.
\end{abstract}

Keywords: gravity surface waves; wave-makers; water-wave generation; computational fluid dynamics

\section{Introduction}

The engineering of surface gravity waves in water channels is a challenging problem in both numerical and experimental setups. The importance of the controlled and efficient generation of waves with prescribed amplitude and phase lies not only in the fundamental research on wave propagation and wave instabilities [1,2], but also in practical applications in hydraulics. For instance, in the recreation of tsunami-wave dynamics in laboratory-scale systems, the employed wave-generation technique plays an important role in the resulting tsunami waveform and dynamics [3,4]. Thus, implementing an efficient source model that is also a realistic representation of a natural wave source is a relevant issue. Another example of the importance of realistic and efficient wave sources comes from the field of fluid-structure interaction $[4,5]$ in experimental and numerical tests of ships and structures interacting with regular waves in the sea [6]. The characterisation of mechanical fatigue in the hull of a ship subjected to multiple collisions with waves is fundamental to design norms and to develop new technologies in shipbuilding $[7,8]$.

Hybridised, mesh-free and finite methods are widely used as a numerical tool for the prediction of the structural response of floating structures to waves [5-7]. Among the Lagrangian mesh-free particle methods, smoothed particle hydrodynamics (SPH) has gained great interest [6,7,9-13]. In such a method, the absence of a discretisation mesh allows to perform otherwise complex and numerically expensive calculations that naturally occur in systems with high deformations, such as shock waves [14-16], high vorticity flows [17], jets [18], drops [19,20], bubbles [21], and spraying [22]. The fluid phase is discretised into a distribution of small fluid particles, whose dynamics are influenced by neighbouring particles that lie within a support domain. For a given particle, the effect of 
the surrounding fluid is determined by a weighted sum over all the remaining particles in the system. Although the SPH method has some drawbacks and numerical issues, such as the well-known tensile instability at low Reynolds numbers [19,23], the method can handle splash and violent, free-surface events. For this reason, the SPH method has become widely used in numerical studies of the interactions of ships and structures with sea waves.

To generate surface waves in water, the usual technique is the inclusion of a wavemakeran oscillating paddle attached to a wall at one of the boundaries of the fluid domain. Such a technique is proven to be very efficient in generating short-wavelength surface waves and is included in mesh-free particle methods to obtain realistic simulations of floating objects interacting with a train of severe waves [6]. Wavemakers can also be used to cancel incident waves, the so-called water-wave active absorbers [24,25], and have shown better performance than passive methods, such as beaches or meta-materials [26]. However, the use of wavemakers has side effects that may represent a drawback in some situations. According to the fundamental theory of wavemakers [27], the oscillations of the wall generates two kinds of waves: a short-range evanescent wave and a long-range radiative wave. Therefore, to provide full control of the amplitude and phase of the generated waves, the region of study must be placed far enough from the wavemaker to avoid nondesired effects from the evanescent wave. Numerical simulations and experiments using this strategy require a large fluid domain, which is costly in numerical operations and experimental resources. Indeed, many previous experimental studies of tsunamis and rogue waves required large water tanks with huge paddles and parts (see Refs. [28-31] for some examples). A way to circumvent this issue in numerical setups is to solve the hydrodynamic equations in regions with different resolutions: a low-resolution fluid domain where waves are generated by the paddles and travel through a long distance, so that evanescent waves decay; and a high-resolution domain, or zone of interest, where the structure is placed in interactions with the incident waves. However, this strategy carries another drawback, which is the energy dissipation intrinsic to the SPH method. A long low-resolution domain also provides dissipation of the radiative wave, thus requiring highenergy of oscillations at the wave paddle. Moreover, paddle wavemakers present another drawback in some numerical and experimental situations, which is their poor performance in the generation of long-wavelength waves: the paddle must oscillate with very high amplitude to produce a long-wavelength wave with a relatively small amplitude [32].

In this article, we propose a new technique for the generation of surface waves in a water channel: the pedal-wavemaking method. The method consists of moving the floor of the channel in a pedalling-like elliptical motion prescribed by the Airy inviscid theory of deep-water waves. Through this technique, we can design waves that emulate deep gravity waves. We demonstrate numerically and theoretically that the technique can generate longwavelength surface-waves, which are elusive for systems with wavemakers. Moreover, since the wave generation technique does not produce an evanescent surface wave, the technique does not require a large fluid domain to produce controlled waves.

The article is organised as follows. Section 2 gives a summary of the wave-generation techniques that are relevant in our study: those by paddle wavemakers and pedal wavemakers. We also give the mathematical model to study wave generation phenomena in a finite-depth tank with a pedalling-like moving bottom. Section 3 shows the results from SPH simulations of regular wave generation. We provide a qualitative comparison between both methods and the Airy theory of deep water waves. We also show in this section the results from the theoretical model of pedal wavemakers. We discuss our results in Section 4. Conclusions and final remarks are given in Section 5.

\section{Methods}

\subsection{Wave Generation Using a Hinged-Paddle with SPH Simulations}

The first method of wave generation considered in this article is the well-known hinged-paddle technique. Figure 1 shows the tow-tank model, so-called as it is of the form used to measure the response of a model ship being towed through the waves. The 
tow-tank has a typical configuration of a hinged-paddle wavemaker for generating waves, and a beach for the dissipation of the waves [33,34]. A two-dimensional layer of inviscid water is contained in a numerical tank of length $L=520 \mathrm{~m}$ and depth $h=40 \mathrm{~m}$. A rigid floor-hinged paddle wavemaker is placed at one end and a gently sloping beach is placed at the other end to reduce reflections. We choose the wavemaker amplitude and frequency to give a wave with wavelength $\lambda=60 \mathrm{~m}$ and amplitude $\eta=2 \mathrm{~m}$ close to the paddle.

We performed numerical simulations of the hydrodynamic equations, using the smoothed particle hydrodynamics (SPH) method $[10,12,13]$. In the SPH method, the field variables and gradients are obtained in a Lagrangian framework through an interpolating kernel - the so-called kernel approximation - that gives a smooth estimate of the physical properties of the fluid, such as the density, pressure, and velocity. The formal space discretisation of hydrodynamic equations is obtained through a particle approximation that estimates smoothed integrals as a sum on a set of fluid particles. We have used a cubic spline smoothing function with a smoothing length of $h_{w}=1.8 D$, with $D$ the diameter of the fluid particles. Finally, time integration is achieved, using standard time-integration methods, such as Runge-Kutta schemes and predictor-corrector methods. We have used $N=4785$ fluid particles to simulate the system of Figure 1. The sea-bottom, the sloping beach and the hinged paddle are shell elements with a prescribed velocity value: timedependent for the latter and fixed for the earliest. The shell elements and SPH particles interact through industry-common contact-interface algorithms.

In Section 3.1, we show that paddles are inefficient in generating long waves and creates evanescent waves on its vicinity. Moreover, although we are considering, in theory, an inviscid fluid, simulations exhibit viscous decay due to numerical effects inherent to the SPH method. To circumvent these issues, we have developed a new wave generation technique described in Section 2.2.

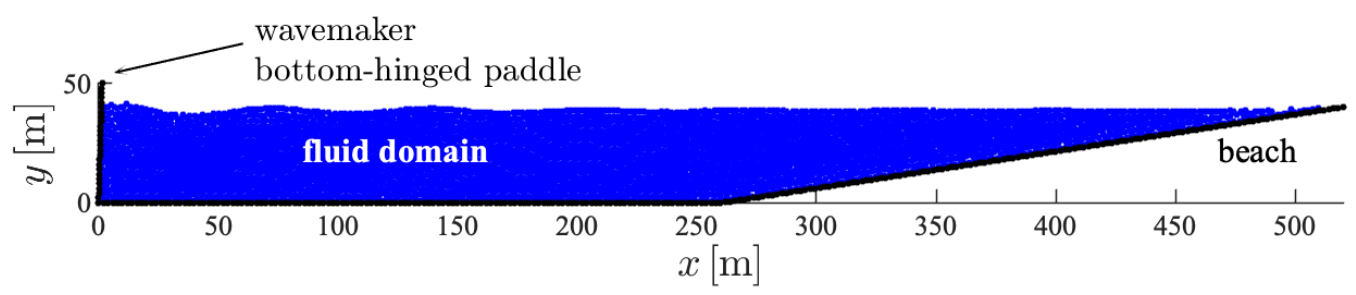

Figure 1. Numerical setup for the tow-tank model. Waves are generated by a hinged-paddle wavemaker placed at the left boundary of a two-dimensional fluid layer of depth $h=40 \mathrm{~m}$ and length $L=520 \mathrm{~m}$. A beach is placed at the right boundary to reduce reflections.

\subsection{The Pedal-Wavemaking Technique}

Waves in fluids are naturally generated by pressure perturbations applied either in the free surface of the water or in the bottom layer. Although such techniques of wave generation are not commonly used in experimental situations, the underlying mechanism occurs naturally in the sea. For instance, waves in the sea are mainly a consequence of wind and atmospheric forcing. Such forcing makes surface fluid particles describe periodiccircular or elliptical - trajectories [35], such as those depicted in Figure 2a. The collective motion of fluid particles at the surface is part of the generated travelling surface wave. For deep-water waves, the response of fluid particles below the surface is given by the Airy linear-wave theory [36]. Particles in the bulk follow the path of circular orbits whose radii decrease exponentially as we go deeper into the fluid.

To develop a new alternative technique for wave generation, we considered the inverse problem: can we generate gravity waves in the free surface, imposing small circular orbits of fluid particles in the bulk? Instead of considering an infinitely deep water-wave system, we considered a reduced subdomain - the wave tank—shown in Figure 2a. The floor of the subdomain is considered to be a boundary with some prescribed motion. Using periodic boundary conditions at the left and right boundaries of the wave tank, orbits in infinitely 
deep water channels can be recreated in this small sub-domain, using moving boundary conditions at the bottom, namely, a pedal-wavemaking motion.

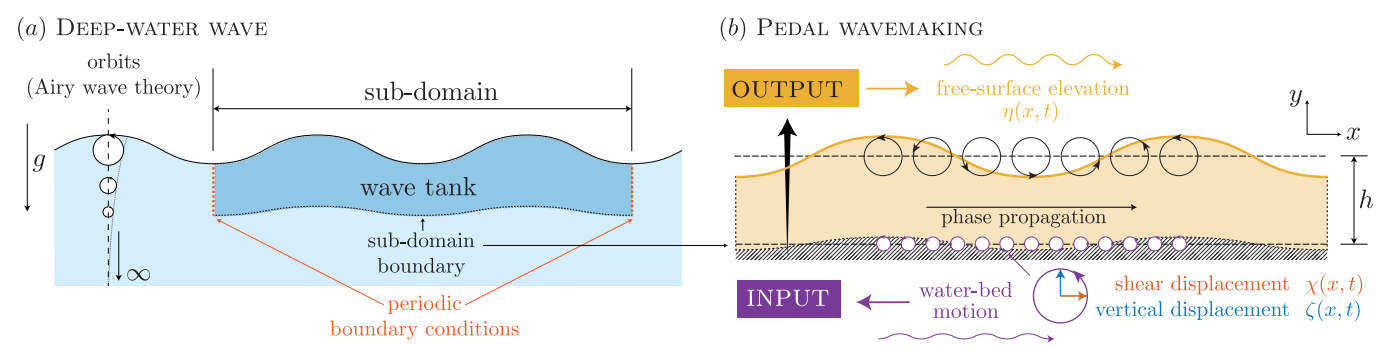

Figure 2. (a) Schematisation of the sub-domain (wave tank) in infinitely deep water. The boundary motion at the bottom of the wave tank is prescribed by the Airy wave inviscid theory. (b) Illustration of the pedal-wavemaking technique. The water bed motion is given by a pedalling-like motion, with horizontal and vertical displacements prescribed by the Airy wave theory at depth $y=-h$. This motion of the water bed gives rise to a travelling wave in the free surface.

Figure $2 \mathrm{~b}$ illustrates our pedal-wavemaking technique in a wave tank of depth $h$. In the SPH formulation, we divided the bottom boundary into small shell elements with prescribed individual motions, according to the Airy wave conditions at the location of each shell. This motion of the individual elements can be regarded as an underwater multipoint mechanism that introduces energy to the fluid. The boundary conditions assigned to these moving shell elements at $y=-h$ are elliptical orbits, which can be decomposed into horizontal and vertical displacements $\chi(x, t)$ and $\zeta(x, t)$, respectively. Thus, the elliptical trajectories of the particles at the bottom of the wave tank are introduced by moving the bottom itself with a given orbital trajectory, resembling a pedalling-like motion.

As we will see in Section 3.2, the pedalling wavemakers efficiently generate longwaves. Notice that the generation of gravity waves by pedal wavemakers, i.e., through the bed motion, is similar to tsunami generation in which long waves are generated by the sudden uplift of the marine base during earthquakes [37]. Tsunamis lie in the longwavelength spectrum of oceanic waves [38]. The pedal-wavemaking technique is, thus, the natural way to generate waves in the region of long wavelengths of the spectrum.

\subsection{Theoretical Model}

A full theoretical approach to the pedal-wavemaking technique, considering all the features of a Newtonian fluid and the right boundary conditions, is required. As we will see in Section 3.2, the fluid is effectively viscous in SPH simulations due to the artificial viscosity inherent to the SPH method. Consequently, we regard the two-dimensional system of Figure $2 \mathrm{~b}$ as an infinitely long channel filled with an incompressible viscous liquid up to a uniform depth $h$. The equations that describe the fluid are given by the Navier-Stokes and incompressibility equation,

$$
\begin{gathered}
\partial_{t} \mathbf{u}+(\mathbf{u} \cdot \nabla) \mathbf{u}=-\frac{1}{\rho} \nabla P+v \nabla^{2} \mathbf{u}-g \hat{\mathbf{y}} \\
\nabla \cdot \mathbf{u}=0,
\end{gathered}
$$

where $\mathbf{u}=(u, v)$ is the velocity field, $P$ is the pressure, $g$ is the acceleration of gravity, $v$ is the kinematic viscosity, and $\rho$ is the density of the fluid.

We decompose the water-bed motion into a vertical and horizontal periodic displacement $\zeta(x, t)$ and $\chi(x, t)$, respectively. A pedalling-like motion of points in the water bed with angular frequency $\omega$ can be described by giving the following appropriate phase difference:

$$
(\chi, \zeta)=\Re\left(i x_{b} e^{i(k x-\omega t)}, y_{b} e^{i(k x-\omega t)}\right),
$$


where $x_{b}$ and $y_{b}$ are complex amplitudes. The collective motion of points in the bottom generates a travelling-wave-like motion of the water bed, i.e., a phase propagation in the positive $x$-direction with wavenumber $k$. The output in the free-surface elevation is a gravity-wave response with amplitude $\eta$.

\section{Results}

\subsection{SPH Simulations of Regular Wave Generation by Hinged Paddles}

Our SPH numerical simulations, using the well-known method of hinged-paddles for wave generation (see Section 2.1), employed a tow-tank model as shown in Figure 1. Our results show that the wave paddle generates a travelling surface wave with wavelength $\lambda \simeq 67 \mathrm{~m}$, as depicted in Figure 3a. However, as the wave propagates towards the beach, its amplitude is significantly reduced due to numerical viscosity. From Figure $3 a$, we notice that near the hinged-paddle, the wave is approximately $2 \mathrm{~m}$ in amplitude, whereas near the beach, it is below a half meter in amplitude. This observation is confirmed in Figure $3 b$, where we show the normalised wave amplitude as a function of the distance from the wavemaker. It is important to remark that the observed decay can be due not only to the physical viscosity, but also to nonphysical energy losses at each of the many numerical smoothing calculations performed as the wave propagates through the tank [39]. The latter is our case in Figure 3, which have artificial viscosity in an otherwise inviscid fluid. Since the object under study, in interaction with waves, must be placed away from the paddle to avoid the disturbances from the evanescent waves, such decay in the wave amplitude becomes a problem.

(a)

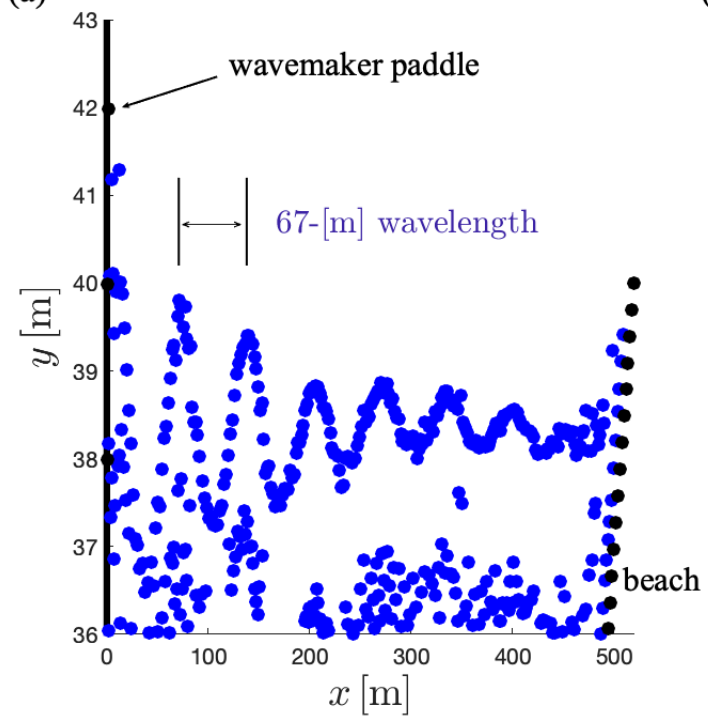

(b)

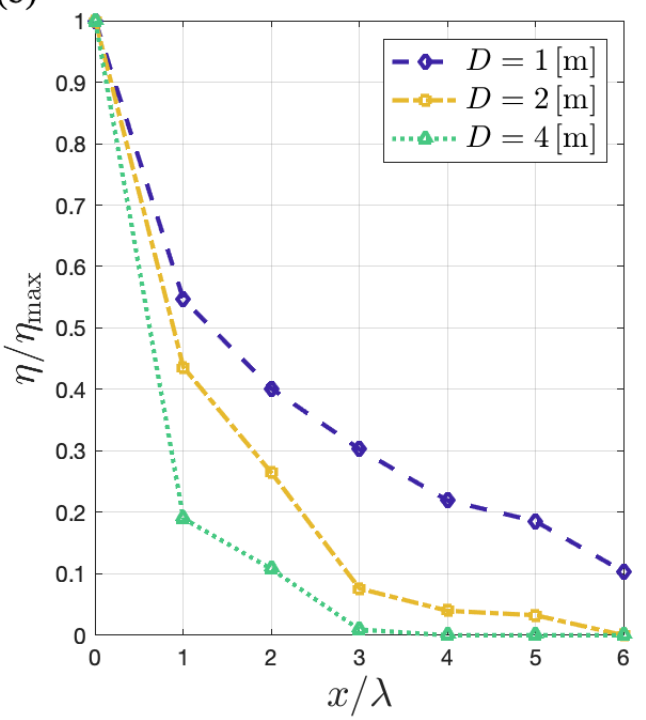

Figure 3. Results from SPH simulations of the tow-tank model. (a) Fluid particles in the SPH simulations, showing that the wave amplitude from the full-size wave tank decreases with distance from the wavemaker. (b) Normalised wave amplitude as a function of the distance from the wavemaker for $\lambda=60 \mathrm{~m}$ and fluid particles with the indicated diameters. Waves generated with small-diameter particles exhibit less energy dissipation. The simulation was run for $120 \mathrm{~s}$, which corresponds to approximately 20 periods of the wavelength.

McCue and co-workers [39] showed, in similar numerical setups, that smaller SPH particles provide less energy dissipation. Following their ideas, we used fluid particles with different diameters $D$. Figure $3 b$ shows the normalised wave amplitude obtained for three diameter values of $\mathrm{SPH}$ particles. Indeed, less energy dissipation is observed for the smallest particles, agreeing with the findings of $\mathrm{McC}$ ue and coworkers [39]. However, the results are still unacceptable for some applications requiring uniform wave amplitudes, such as in the study of ship motion in waves [7]. To limit wave-amplitude loss to around 
$10 \%$ over 6 wavelengths would require $D \sim 0.1 \mathrm{~m}$ [7]. The use of such small particles in a $260 \mathrm{~m}$-length wave tank would dramatically increase the computational cost. Other authors $[40,41]$ suggested an improved time-stepping algorithm as a possible solution to reducing the wave decay. De Padova and coworkers [42] also showed high dissipation in their generated waves in a tank with a sloping floor, including both regular and irregular waves. Such a configuration ultimately led to breaking on a shore, so even those waves were not directly comparable to a constant-depth wave tank for ship-motion predictions. De Padova and coworkers concluded that a small value of one of the empirical coefficients of the SPH artificial viscosity was required for numerical stability, but the result was still too dissipative for accurate wave reproduction.

Another well-known important drawback of the hinged-paddle wavemaker is its poor performance in the generation of long-wavelength waves. Let us define the efficiency of the wavemaker as follows:

$$
\varepsilon:=\frac{a(h / \lambda)}{s},
$$

with $s$ as the displacement of the paddle and $a$ as the amplitude of the radiative wave, which is a function of the ratio $h / \lambda$. Here, $h$ is the depth of the channel and $\lambda$ is the wavelength $[32,33]$. The following can be shown in the short-wavelength limit:

$$
\lim _{h / \lambda \rightarrow \infty} \varepsilon=1,
$$

which means that the amplitude of the wavemaker's oscillations must be of the order of the wave amplitude, i.e., $s \sim a$. However, the efficiency decays as $h / \lambda$ in the long-wavelength limit $h / \lambda \rightarrow 0$, which means that the wavemaker must oscillate at an amplitude of the order of the wavelength, i.e., $s \sim a \lambda / h$. Thus, the paddle must oscillate with a very high amplitude to produce a long-wavelength wave with a relatively small amplitude. Consequently, the generation of waves by replicating the wave tank physics, using paddletype wavemakers in the SPH formulation, is not a viable approach to predict the response of structures to long waves.

\subsection{Generation of Long Waves by Pedal Wavemakers}

The pedal-wavemaking technique introduced in Section 2.2 does not show any wave decay or evanescent waves near the source and is able to generate long-wavelength waves as we will show in the following. Figure 4 depicts typical long waves generated with the pedal-wavemaking method. In Figure $4 \mathrm{a}$, we generate a wave with $\lambda=L_{w w}$, with $L_{w t}=2.5 \mathrm{~m}$ the length of the wave-tank; in Figure $4 \mathrm{~b}$, we produce a wave with $\lambda=60 \mathrm{~m}$. In both cases, the waves are obtained with the pedal-wavemaking technique, using $\mathrm{SPH}$ particles with diameter $D=2[\mathrm{~m}]$.

Notice that there is no loss of wave amplitude and there are no evanescent surface waves throughout the wave tank in Figure $4 \mathrm{~b}$. This is an intrinsic consequence of the forcing method: the pedal-wavemakers at the bottom drive the fluid simultaneously throughout the whole length of the water channel, thus sustaining an extended wave in the fluid domain. However, it is important to remark that the pedal-wavemaking technique does not overcome the losses inherent to the SPH formulation. The pedal wavemaking relies on the interaction of a wave with the floor in shallow depths.

In the simulations of Figure 4, the wave is developed simultaneously everywhere throughout the tank in response to the pedalling motion on the floor. Thus, fully developed waves rapidly fill the wave tank. Waves, due to the pedalling motion, are injected throughout the seabed and not at one extreme of the tank, as is the case for a single hingedpaddle. We observed that for some optimal values of the frequency and wavelength, a small pedalling motion in the seabed can generate a relatively high-amplitude gravity wave. Figure $4 \mathrm{~b}$ shows a $2 \mathrm{~m}$ amplitude surface wave designed with $\lambda=60 \mathrm{~m}$ and frequency $f=0.8 \mathrm{~Hz}$. We used SPH particles with diameter $D=1 \mathrm{~m}$, and the floor of the wave tank was divided into eight equal segments with a $0.88 \mathrm{~m}$ diameter pedalling-like motion. 
The amplitude of the generated wave was greater than the diameter of the pedalling by a factor greater than two. This observation suggests that the system is near some resonance condition at the given wavelength and frequency. Further studies characterising such resonance will be published elsewhere.
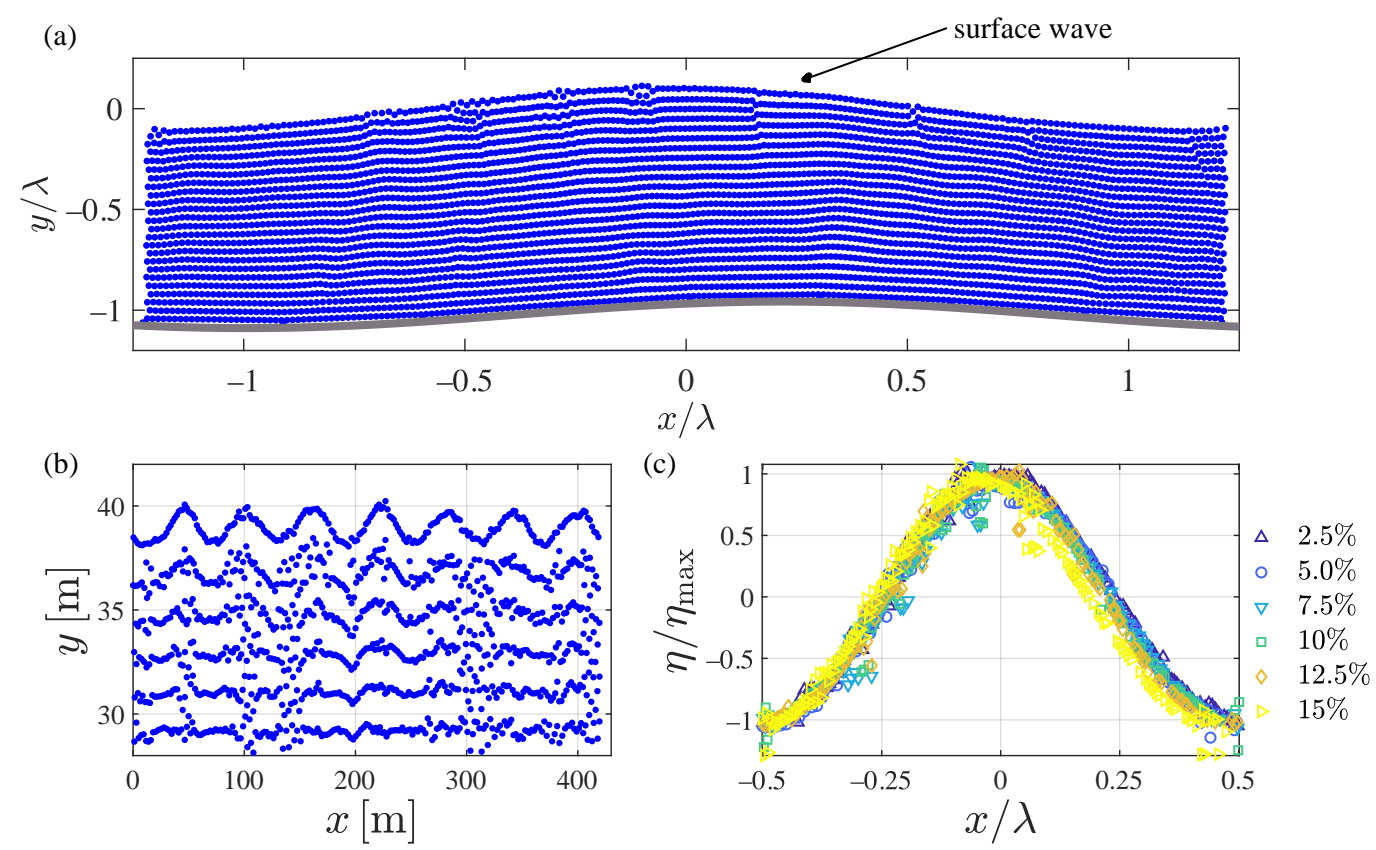

Figure 4. SPH simulations of long-wavelength generation, using the pedal-wavemaking technique. (a) A typical, fully developed long-wavelength wave. (b) Wave generated with $\lambda=60 \mathrm{~m}$ and $f=0.8 \mathrm{~Hz}$ by pedal-wavemaking. A pedalling-like motion of diameter $0.88 \mathrm{~m}$ at the bottom generates a $2 \mathrm{~m}$ amplitude surface wave. (c) Normalised profiles of surface waves obtained in wave-tanks of different depths-see explanation in the text. The observed wave amplitude is $2 \%$ of the wavelength. The roughness of the surface is due to the discretisation of the fluid into particles. Each profile has been normalised by its actual wave amplitude $\eta$.

To characterise how the depth of the wave-tank affects the properties of waves generated through pedal-wavemaking, let us introduce the following dimensionless variables:

$$
\tilde{\eta} \equiv \frac{\eta}{\lambda}, \quad \tilde{h} \equiv \frac{h}{\lambda}, \quad \tilde{D} \equiv \frac{D}{\lambda} .
$$

From the simulation shown in Figure $4 b$, one obtains $\tilde{\eta} \simeq 0.03$, which is in accordance with the long-wave regime. We performed SPH simulations, using different depths in the range $0.025 \leq \tilde{h} \leq 0.15$. Normalised surface profiles at a fixed time are shown in Figure $4 \mathrm{c}$ for the indicated values of the depth. For all the floor depths under study, we noticed that the profile of the wave seems to be unchanged. This suggests that our wave-generation technique, using pedal wavemakers, is robust to the depth of the wave-tank, and thus, it emulates deep-water waves under controlled situations.

Pedal wavemakers can be used solely to generate and sustain travelling waves, as we show in the three-dimensional numerical model of Figure 5. A ship model with appropriate degrees of freedom can be placed in interaction with waves in a wave tank. In Figure 5, we generate a $1.5 \mathrm{~m}$ height travelling wave, using only four shell elements per wave for the water bed. The nodes of such elements were prescribed with time-dependent boundary conditions to enable pedal-wavemaking floor motions. As the ship moves forward, there will be oblique and transverse, ship-induced waves with some wavelength $\lambda_{s}$. These waves will propagate independent of the dominant pedal-motion generated waves, and may dissipate due to interaction with the bottom under shallow-water conditions. Notice from 
Figure 5 that the maximum horizontal velocity is located in the bulk of the fluid, just below the surface. We will come back to this point in Section 3.3.

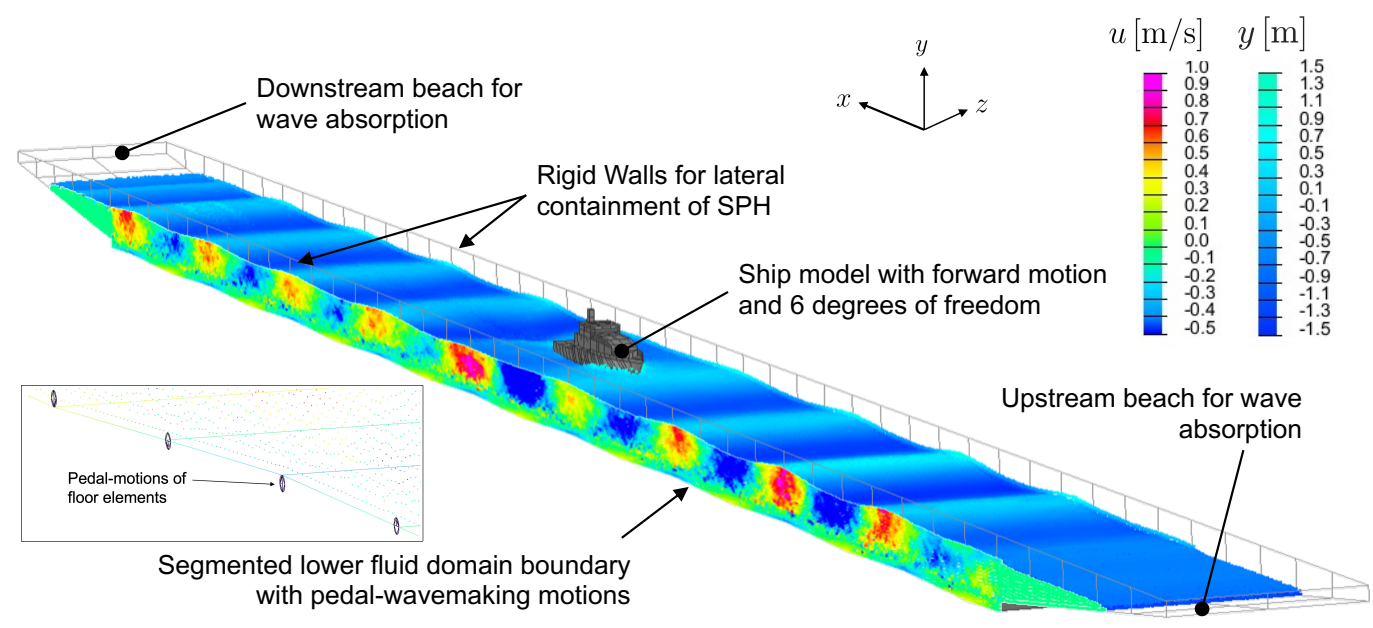

Figure 5. Visual representation of a three-dimensional numerical model of a floating rigid ship interacting with waves. A $1.5 \mathrm{~m}$ wave is generated and sustained, using only the pedal-wave making floor motions.

\subsection{Emulating Deep-Water Waves in a Wave Tank}

Figure 6 shows the through-depth velocity profiles in the horizontal and vertical directions for $\tilde{h}=0.10$. We have verified that numerical results are qualitatively similar for any wave-tank depth. The horizontal and vertical velocity profiles, according to the Airy inviscid deep-water-wave theory, are shown in Figure 6a,b, respectively, for the given values of the parameters. According to the deep-water-wave theory, the amplitude of the wave decreases exponentially with the depth as $\exp (k y)$, where $y$ is the vertical coordinate and $k=2 \pi / \lambda$. The horizontal and vertical components of the velocity can be easily obtained knowing the frequency of the oscillations of the wave, namely $\omega=2 \pi f$. Let $u$ and $v$ be the horizontal and vertical components of the velocity of a fluid particle placed at an average depth $y_{p}$, respectively. Thus, according to the deep-water-wave theory, trajectories of fluid particles in phase spaces $\{(u, y)\}$ and $\{(v, y)\}$ are as follows:

$$
\begin{gathered}
y=y_{p}+\left.\Delta y\right|_{y=0}\left(\frac{u}{\left.u\right|_{y=0}}\right), \\
\left(\frac{y-y_{p}}{\left.\Delta y\right|_{y=0}}\right)^{2}+\left(\frac{v}{\left.v\right|_{y=0}}\right)^{2}=\exp (2 k y),
\end{gathered}
$$

which corresponds to straight lines and ellipses for the vertical and horizontal components of velocity, respectively, as depicted in Figure 6c. From Equation (6a), it follows that the slope of $y(u)$ is positive and increases in absolute value with the wavelength. Likewise, from Equation (6b), it follows that the area enclosed by the elliptical trajectories decreases with the depth.

Figure $6 \mathrm{~d}$,e shows the horizontal and vertical velocity profiles obtained from numerical simulations in SPH, using the pedal-wavemaking technique. The similarities with the corresponding profiles from the deep-water-wave theory are remarkable. However, Figure $6 \mathrm{~d}$ reveals that the maximum values of the horizontal velocity are located below the surface, which is slightly different from the predictions of the inviscid Airy theory. On the contrary, the vertical velocity field is in good agreement with the deep-water velocity profile. Trajectories of SPH particles have also shown deep-water-wave-like behaviour, as depicted in Figure 6f. Trajectories in the vertical component describe ellipses in good 
agreement with the deep-water theory. However, trajectories in the horizontal component exhibit slight deviations from the Airy theory.

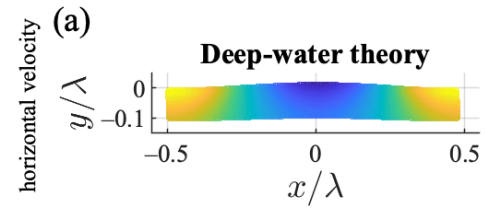

(b)

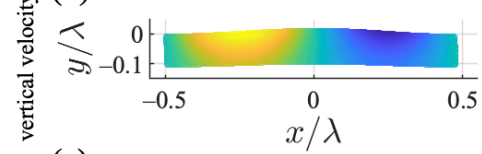

(c)

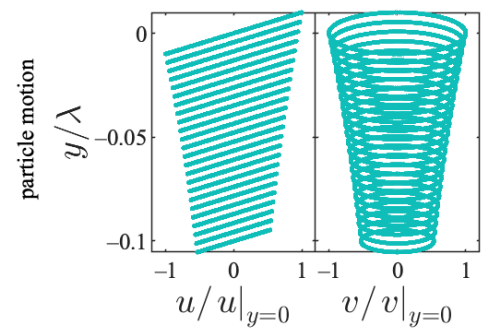

(d)

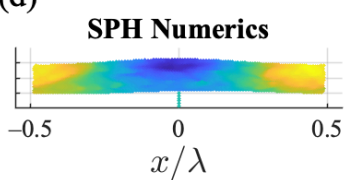

(e)

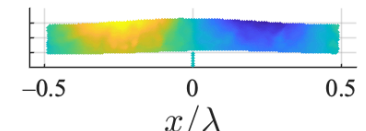

(f)

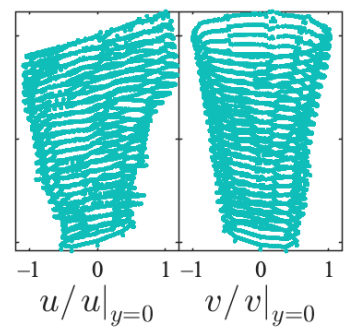

(g)

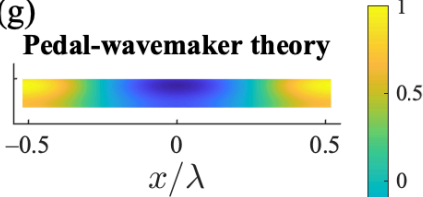

(h)

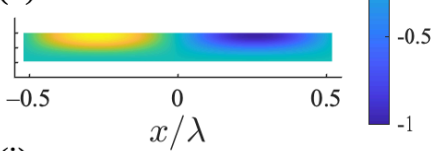

(i)

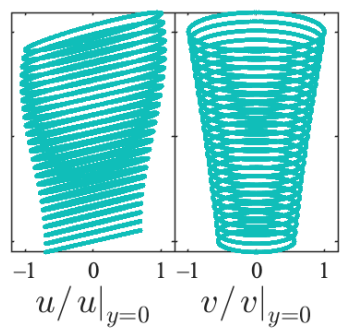

Figure 6. Comparison between the Airy theory for inviscid deep water waves $(\mathbf{a}-\mathbf{c})$, SPH numerical simulations, using pedal wavemakers at a depth of $10 \%$ of the wavelength $(\mathbf{d}-\mathbf{f})$, and the theory of pedal wavemakers with $\bar{\theta}=10^{-1}$ and $\bar{\lambda}=10$ (g-i). The diameter of the pedalling-like motion is 0.01 $\mathrm{h}$. The upper row shows typical profiles of the horizontal velocity, whereas the central row shows the corresponding vertical velocity profiles. The colour scale indicates the normalised value of the speed. The normalised vertical and horizontal velocity profiles of fluid particles within the wave are depicted in the lower row.

\subsection{Linearised Hydrodynamic Equations in the Long-Wavelength Limit}

From the numerical simulations of the previous section, we conclude that waves generated by pedal wavemakers can emulate deep-water waves in a finite-depth tank. However, we observed some deviations from the Airy theory in the horizontal components of the velocity field. This mismatch is related to the effect of viscosity and the formation of boundary layers at the bottom and below the surface, as will be shown.

To solve the linear version of the system (1), we used the Helmholtz decomposition $\mathbf{u}=\boldsymbol{\nabla} \phi+\boldsymbol{\nabla} \times(\psi \hat{\mathbf{z}})$, where $\phi$ is the velocity potential and $\psi$ is the stream function, with $\hat{\mathbf{z}} \equiv \hat{\mathbf{x}} \times \hat{\mathbf{y}}$ [36]. Thus, the linearised system (1) decouples $\phi, \psi$ and $P$ in the bulk of the fluid via the following three equations:

$$
\begin{gathered}
\nabla^{2} \phi=0, \\
\partial_{t} \psi-v \nabla^{2} \psi=0, \\
\partial_{t} \phi+\frac{P}{\rho}+g y=0 .
\end{gathered}
$$

The linearised boundary condition at the top interface yields the following:

$$
\begin{gathered}
\partial_{t} \eta-\partial_{y} \phi+\left.\partial_{x} \psi\right|_{y=\eta}=0, \\
2 v\left(\partial_{y y} \phi-\partial_{x y} \psi\right)-\left.\frac{P}{\rho}\right|_{y=\eta}=0, \\
2 \partial_{x y} \phi-\partial_{x x} \psi+\left.\partial_{y y} \psi\right|_{y=\eta}=0 .
\end{gathered}
$$


Equation (8a) is the kinematic condition, whereas Equations (8b) and (8c) are the conditions for the normal and tangential stresses, respectively. At the bottom of the wave tank, we assume the following no-slip boundary conditions:

$$
\begin{aligned}
& \partial_{t} \chi-\partial_{x} \phi-\left.\partial_{y} \psi\right|_{y=-h}=0, \\
& \partial_{t} \zeta-\partial_{y} \phi+\left.\partial_{x} \psi\right|_{y=-h}=0 .
\end{aligned}
$$

The system of Equation (7) together with the boundary conditions of Equations (8) and (9) determines the linear response of the system. The general solutions of the bulk Equations (7a) and (7b) are as follows:

$$
\begin{aligned}
\phi(x, y, t) & =\frac{\omega}{k} \Re\left[(A \cosh k y+B \sinh k y) e^{i(k x-\omega t)}\right], \\
\psi(x, y, t) & =\frac{\omega}{k} \Re\left[(C \cosh m y+D \sinh m y) e^{i(k x-\omega t)}\right],
\end{aligned}
$$

with $m^{2}=k^{2}-i \omega / v$. The values of the complex constants $(A, B, C, D)$ are determined by the boundary conditions. Herein, it is convenient to introduce the following dimensionless quantities:

$$
\bar{k} \equiv k h, \quad \bar{m} \equiv m h, \quad \bar{\lambda} \equiv \frac{\lambda}{h}, \quad \bar{\omega} \equiv \frac{\omega}{\omega_{\infty}},
$$

where the characteristic frequency $\omega_{\infty}$ is given by the dispersion relation for deep-water waves, i.e., $\omega_{\infty}^{2}=g k$. Let $\bar{\theta} \equiv 2 v k^{2} / \omega$ be the dimensionless viscosity and $\alpha \equiv m / k$ be the ratio between the wave numbers $m$ and $k$. Notice that $\bar{\theta}$ could also be interpreted as a squared comparison between the extent of oscillatory Stokes boundary layers, $\sqrt{2 v / \omega}$, and the wavelength $\lambda$. Evaluating the bulk solutions (10) in the system (7) and normalizing the spatial coordinates according to $\bar{x} \equiv x / h$ and $\bar{y} \equiv y / h$, one obtains the following linear system of equations:

$$
\left(\begin{array}{cccc}
\bar{\omega}^{2} \bar{\theta} \beta & i & 1 & -i \bar{\omega}^{2} \bar{\theta} \alpha \\
0 & i & \beta & 0 \\
i \cosh \bar{k} & -i \sinh \bar{k} & -\alpha \sinh \bar{m} & \alpha \cosh \bar{m} \\
-i \sinh \bar{k} & i \cosh \bar{k} & \cosh \bar{m} & -\sinh \bar{m}
\end{array}\right)\left(\begin{array}{c}
\bar{A} \\
\bar{B} \\
\bar{C} \\
\bar{D}
\end{array}\right)=\left(\begin{array}{c}
0 \\
0 \\
\bar{x}_{b} \\
\bar{y}_{b}
\end{array}\right)
$$

with $\beta \equiv 1-i / \bar{\theta}, \bar{x}_{b} \equiv x_{b} / h$ and $\bar{y}_{b} \equiv y_{b} / h$. Solving Equation (12) for the dimensionless vector $(\bar{A}, \bar{B}, \bar{C}, \bar{D})^{T} \equiv(A, B, C, D)^{T} / h$, one obtains from Equation (10) the following velocity potential and stream functions:

$$
\begin{gathered}
\phi(x, y, t)=\frac{\omega}{k} \Re\left\{\left[\frac{i \alpha \bar{\theta}}{\Gamma_{B}^{(0)}(\bar{\theta}-i)}\left(\mathcal{F}_{p}+\frac{\left(\alpha \bar{\theta}^{2} \bar{\omega}^{2} \Gamma_{B}^{(1)}-\Gamma_{B}^{(0)}-\Gamma_{S}^{(0)}\right) \mathcal{P}}{\alpha^{2} \bar{\theta}^{2} \bar{\omega}^{2}\left(\Gamma_{S}+\bar{\theta} \Gamma_{B}\right)}\right) \cosh (k y)\right.\right. \\
\left.\left.+\frac{(1+i \bar{\theta}) \mathcal{P}}{\alpha \bar{\theta}\left(\Gamma_{S}+\bar{\theta} \Gamma_{B}\right)} \sinh (k y)\right] e^{i(k x-\omega t)}\right\}, \\
\psi(x, y, t)=\frac{\omega}{k} \Re\left[\frac{\mathcal{P} e^{m y}}{\alpha\left(\Gamma_{S}+\bar{\theta} \Gamma_{B}\right)} e^{i(k x-\omega t)}\right] .
\end{gathered}
$$

The constants $\mathcal{F}_{p}$ and $\mathcal{P}$ in Equation (13), related to the pedal-wavemaking motion at the bottom, are given by the following:

$$
\begin{gathered}
\mathcal{F}_{p} \equiv x_{b} \sinh \bar{k}+y_{b} \cosh \bar{k} \\
\mathcal{P} \equiv \alpha \bar{\theta} \mathcal{F}_{p}-(\bar{\theta}-i)\left(x_{b} \sinh \bar{m}+\alpha y_{b} \cosh \bar{m}\right) .
\end{gathered}
$$


The constants $\Gamma_{S}^{(0)}$ and $\Gamma_{S}$ are related to the angular frequency, depth, wavenumbers and viscosity through the following:

$$
\begin{gathered}
\Gamma_{S}^{(0)} \equiv(i-\bar{\theta}) \alpha \bar{\theta} \bar{\omega}^{2}, \\
\Gamma_{S} \equiv(i-\bar{\theta})\left(1+\alpha \bar{\theta}^{2} \bar{\omega}^{2}\right) .
\end{gathered}
$$

Finally, the constants $\Gamma_{B}^{(0)}, \Gamma_{B}^{(1)}$, and $\Gamma_{B}$ are given by the following:

$$
\begin{gathered}
\Gamma_{B}^{(0)} \equiv \alpha \sinh \bar{k} \cosh \bar{m}-\cosh \bar{k} \sinh \bar{m}, \\
\Gamma_{B}^{(1)} \equiv \alpha \sinh \bar{k} \sinh \bar{m}-\cosh \bar{k} \cosh \bar{m}, \\
\Gamma_{B} \equiv \Gamma_{B}^{(0)}-\Gamma_{B}^{(1)}+\sigma(\alpha \cosh \bar{k} \cosh \bar{m}-\sinh \bar{k} \sinh \bar{m}),
\end{gathered}
$$

with $\sigma \equiv \bar{\omega}^{2}(\bar{\theta}-i)^{2}$.

The velocity fields shown in Figure $6 \mathrm{~g}, \mathrm{~h}$ were obtained from Equation (13) and agree well with SPH numerical simulations. Thus, the pedal-wavemaker theory predicts deepwater-like behaviour in the vertical velocity field. Moreover, it also predicts maximum horizontal velocity values below the free surface, due to a top boundary layer, showing that the feature observed in SPH simulations is consistent with the effect of viscosity.

\section{Discussion}

The theoretical predictions for the horizontal and vertical components of the velocity of a fluid particle as a function of $\bar{y}$ for the parameters of the numerical simulation of Section 3.3 are depicted in Figure 6i. Notice that the amplitude in the oscillations of $\bar{u}$ and $\bar{v}$ decays almost linearly with the depth, which is a characteristic behaviour of shallow-water waves [2]. Indeed, we expect a shallow-water profile, given that the wavelength is large, compared to the depth of the wave tank. However, the linear decay in the oscillations of $\bar{u}$ and $\bar{v}$ can be regarded as an exponential decay in a large domain truncated to the first-order in depth. Thus, Figure $6 c, f, i$ can be regarded as the trajectories of fluid particles near the surface of a deep-water wave truncated from below by the wave tank. We conclude that the pedal-wavemaking technique emulates with good accuracy the upper fluid layers of deep-water waves, using a shallow wave tank.

The form of the velocity potential and streaming functions of Equation (13) leads us to conclude that the dynamics of fluid particles depends on the viscosity, depth, angular frequency and the wavelength. For practical purposes, we introduce the dimensionless number $\bar{\Theta} \equiv 2 v / \sqrt{g h^{3}}=\bar{\theta} \bar{\omega} / \bar{k}^{3 / 2}$, which is independent of $\omega$ and $k$. Figure 7 summarises the different type of behaviours obtained through the systematic span of the $(\bar{\lambda}, \bar{\Theta})$-space (we fixed $\bar{\omega}=1$ in all the cases). Figure 7a shows the case of small viscosity, namely, $\bar{\Theta}=10^{-2}$. For $\bar{\lambda}=2$, a boundary layer becomes evident at the bottom and below the surface, resulting in an hourglass-like shape in the collective orbits. In this hourglasslike case, the amplitude of the surface wave is nearly the same as the amplitude of the pedalling motion. The behaviour changes dramatically for $\bar{\lambda}=3$, exhibiting an almost deep-water-like behaviour with a significant gain and a thin boundary layer at the bottom. As the wavelength further increases, the system progressively emulates the upper layers of a truncated deep-water behaviour, which is qualitatively similar to the cases shown in Figure 6.

Figure $7 \mathrm{~b}$ shows the particle orbits for moderate viscosity, namely, $\bar{\Theta}=10^{-1}$. For $\bar{\lambda}=2$, one obtains a configuration in the form of an Aryballos jar with a thick boundary layer at the bottom of the wave tank. Due to viscosity, the amplitude of the surface wave is smaller than the amplitude of the pedalling motion. The boundary layer at the bottom decreases as the wavelength increases. For $\bar{\lambda}=4$, the gain in the amplitude of the surface wave is large, and the collective configuration of orbits shows a rotation of the elliptical axes, exhibiting a tornado-like shape. This feature becomes evident around $\bar{y}=-0.7$, 
where the slope of the principal axis of the orbits reaches a maximum value and changes its sign. For $\bar{\lambda}=10$, the system emulates the upper layers of a truncated, deep-water behaviour with a thin boundary layer at the bottom.
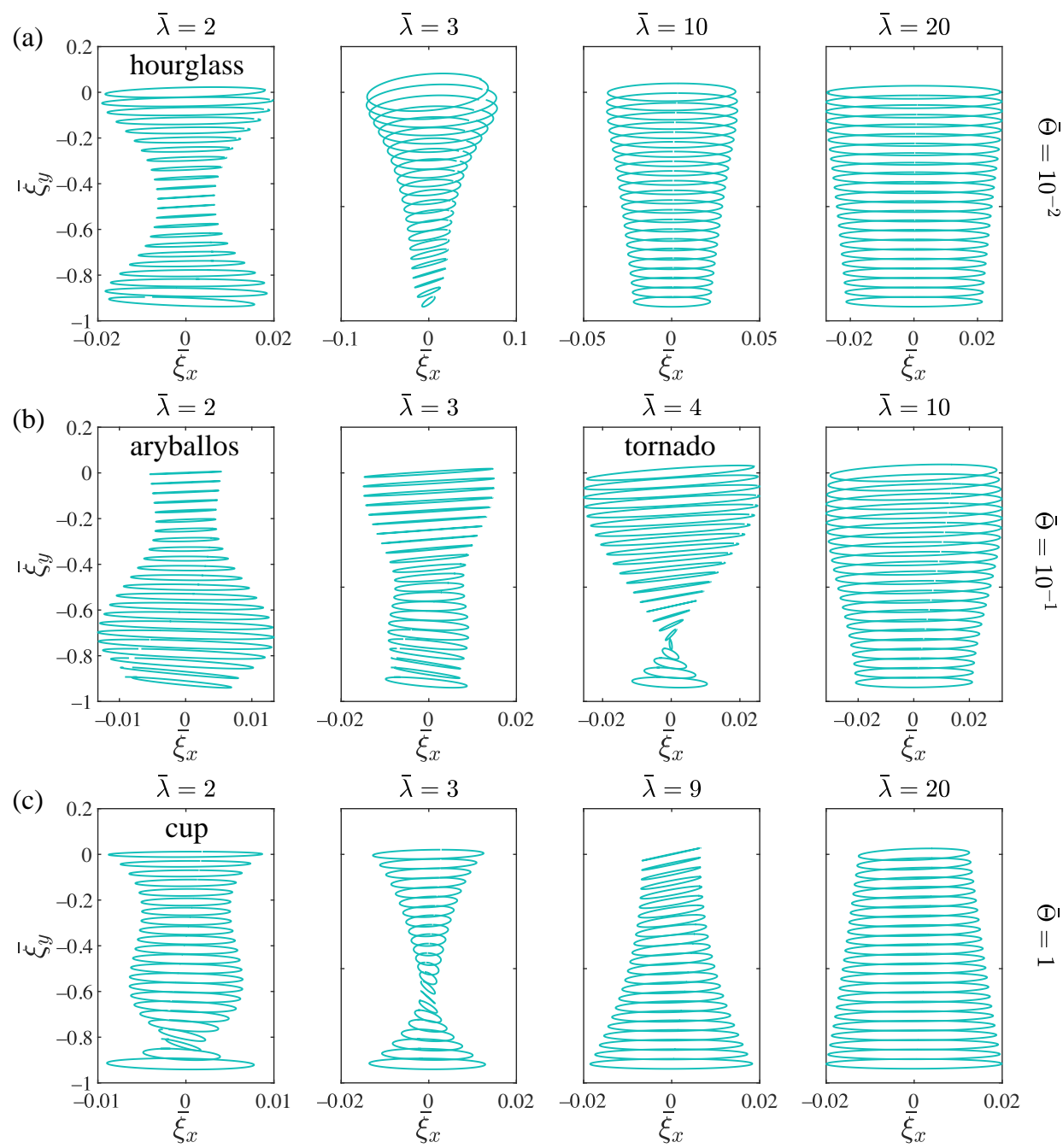

Figure 7. Oscillatory particle excursion $\bar{\xi}_{y}$, using the pedal-wavemaking technique with $\bar{\omega}=1$ and $\bar{x}_{b}=\bar{y}_{b}=1 / 24$ for $(\mathbf{a}) \bar{\Theta}=10^{-2}$, (b) $\bar{\Theta}=10^{-1}$, and (c) $\bar{\Theta}=1$. The normalized wavelength of the generated gravity wave is indicated for each case.

Finally, Figure 7c shows the particle orbits for large viscosity, namely, $\bar{\Theta}=1$. For $\bar{\lambda}=2$, the collective configuration of orbits exhibits a cup-like shape. The slope of the principal axis of the closed orbits reaches a maximum value near the bottom. However, contrary to the tornado-like configuration, there is no change in the orientation of the axes of the ellipses. For $\bar{\lambda}=9$, the system exhibits an almost deep-water-like behaviour with gain smaller than unity due to viscosity. The system emulates deep-water behaviour for larger values of the wavelength.

In summary, from our numerical and mathematical modelling of water waves, we conclude that the pedal-wavemaking technique can emulate deep-water behaviour in a shallow wave tank. Because of viscosity, thin boundary layers appear near the bottom and below the surface, generating vorticity production in such layers and producing a mismatch in the horizontal velocity with inviscid deep-water theory. Notwithstanding, the vertical velocity field, using pedal-wavemakers, is almost identical to the corresponding velocity field in inviscid deep-water waves. 


\section{Conclusions}

We introduced a new technique to recreate deep long gravity waves in finite-depth water channels, using pedal wavemakers at the bottom. The technique consists of moving small segments of the seabed with a periodic motion, resulting in a multi-point, pedal-like action. The underlying mechanism is efficient in the generation of long wavelengths, which has been elusive using conventional wavemakers [32]. The generated wave has no longitudinal loss of wave amplitude, due to artificial dissipation in SPH simulations. Studying the linear response of the water free-surface to the pedal-like motion in the bottom, we obtained results in full agreement with the SPH simulations that emulate deep-water behaviour, according to the linear Airy theory. We demonstrated that the problem of evanescent waves appearing near hinged-paddle wavemakers is converted into a thin boundary layer below the surface and at the bottom, using pedal-wavemaking. No evanescent waves are present on the surface when the waves are generated by the pedal-wavemakers.

Our wave-generation technique gives useful guidelines to recreate deep-wave phenomena in both experimental and numerical setups with minimum resources. Although the present study is focused on single frequency waves, applications may go beyond. Once the linear water-wave generation problem is understood, it is possible to implement a superposition of wave frequencies with different Fourier amplitudes to generate an arbitrary shape of the bottom motion and the surface wave. Moreover, localised, pedal-motion floor excitation can be used to establish localised and directional wave propagation, which may be relevant for the study of transient waves in some applications. More applications may be also found in the study of floating structures interacting with regular waves under realistic oceanic conditions.

Supplementary Materials: The following are available at https://www.mdpi.com/article/10.3390/ fluids6060222/s1.

Author Contributions: Conceptualisation, B.C., L.G. and J.F.M.; formal analysis, I.V., A.L.A., L.G. and J.F.M.; investigation, I.V., B.C., A.L.A., L.G. and J.F.M.; methodology, B.C., L.G. and J.F.M.; software, B.C. and L.G.; supervision, J.F.M.; validation, B.C., L.G. and J.F.M.; visualization, B.C., L.G. and J.F.M.; writing-original draft, J.F.M.; writing—review and editing, B.C. and L.G. All authors have read and agreed to the published version of the manuscript.

Funding: I.V. and L.G. were funded by Fondecyt/Iniciación, grant No. 11170700. B.C. was funded by the Australian Research Council Linkage Project Number LP190101283. J.F.M. was funded by Universidad de Santiago de Chile through the POSTDOC_DICYT, grant number 042031GZ_POSTDOC and ANID FONDECYT/POSTDOCTORADO/3200499.

Institutional Review Board Statement: Not applicable.

Informed Consent Statement: Not applicable.

Data Availability Statement: The data presented in this study are available in the supplementary material.

Conflicts of Interest: The authors declare no conflict of interest. The funders had no role in the design of the study; in the collection, analyses, or interpretation of data; in the writing of the manuscript, or in the decision to publish the results.

\section{References}

1. Cross, M.; Greenside, H. Pattern Formation and Dynamics in Nonequilibrium Systems; Cambridge University Press: Cambridge, UK, 2009.

2. Lighthill, J. Waves in Fluids; Cambridge University Press: Cambridge, UK, 1978.

3. Grilli, S.T.; Harris, J.C.; Bakhsh, T.S.T.; Masterlark, T.L.; Kyriakopoulos, C.; Kirby, J.T.; Shi, F. Numerical simulation of the 2011 Tohoku tsunami based on a new transient FEM co-seismic source: Comparison to far-and near-field observations. Pure Appl. Geophys. 2013, 170, 1333-1359. [CrossRef]

4. Jamin, T.; Gordillo, L.; Ruiz-Chavarría, G.; Berhanu, M.; Falcon, E. Experiments on generation of surface waves by an underwater moving bottom. Proc. R. Soc. A Math. Phys. Eng. Sci. 2015, 471, 20150069. [CrossRef] 
5. Cunningham, L.S.; Rogers, B.D.; Pringgana, G. Tsunami wave and structure interaction: An investigation with smoothed-particle hydrodynamics. Proc. Inst. Civ. Eng. Eng. Comput. Mech. 2014, 167, 126-138. [CrossRef]

6. Groenenboom, P.H.; Cartwright, B.K. Hydrodynamics and fluid-structure interaction by coupled SPH-FE method. J. Hydraul. Res. 2010, 48, 61-73. [CrossRef]

7. Cartwright, B.K. The Study of Ship Motions in Regular Waves Using a Mesh-Free Numerical Method. Master's Thesis, University of Tasmania, Hobart, Australia, 2012.

8. Lloyd, A.R.J.M.; Hosoda, R.; Robinson, D.W.; Nicholson, K.; Victory, G.; Price, W.G.; Bishop, R.E.D.; Price, W.G. Seakeeping. Philos. Trans. R. Soc. London Ser. A Phys. Eng. Sci. 1991, 334, 253-264. [CrossRef]

9. Li, S.; Liu, W.K. Meshfree and particle methods and their applications. Appl. Mech. Rev. 2002, 55, 1-34. [CrossRef]

10. Liu, G.R.; Liu, M.B. Smoothed Particle Hydrodynamics: A Meshfree Particle Method; World Scientific: Singapore, 2003.

11. Sigalotti, L.D.G.; Klapp, J.; Sira, E.; Meleán, Y.; Hasmy, A. SPH simulations of time-dependent Poiseuille flow at low Reynolds numbers. J. Comput. Phys. 2003, 191, 622-638. [CrossRef]

12. Liu, M.B.; Liu, G.R. Smoothed Particle Hydrodynamics (SPH): An Overview and Recent Developments. Arch. Comput. Methods Eng. 2010, 17, 25-76. [CrossRef]

13. Wang, Z.B.; Chen, R.; Wang, H.; Liao, Q.; Zhu, X.; Li, S.Z. An overview of smoothed particle hydrodynamics for simulating multiphase flow. Appl. Math. Model. 2016, 40, 9625-9655. [CrossRef]

14. Sigalotti, L.D.G.; López, H.; Trujillo, L. An adaptive SPH method for strong shocks. J. Comput. Phys. 2009, $228,5888-5907$. [CrossRef]

15. Sigalotti, L.D.G.; López, H.; Donoso, A.; Sira, E.; Klapp, J. A shock-capturing SPH scheme based on adaptive kernel estimation. J. Comput. Phys. 2006, 212, 124-149. [CrossRef]

16. Marrone, S.; Antuono, M.; Colagrossi, A.; Colicchio, G.; Touzé, D.L.; Graziani, G. $\delta$-SPH model for simulating violent impact flows. Comput. Methods Appl. Mech. Eng. 2011, 200, 1526-1542. [CrossRef]

17. Sun, P.; Colagrossi, A.; Marrone, S.; Antuono, M.; Zhang, A. Multi-resolution Delta-plus-SPH with tensile instability control: Towards high Reynolds number flows. Comput. Phys. Commun. 2018, 224, 63-80. [CrossRef]

18. De Padova, D.; Mossa, M.; Sibilla, S. Characteristics of nonbuoyant jets in a wave environment investigated numerically by SPH. Environ. Fluid Mech. 2020, 20, 189-202. [CrossRef]

19. Meleán, Y.; Sigalotti, L.D.G.; Hasmy, A. On the SPH tensile instability in forming viscous liquid drops. Comput. Phys. Commun. 2004, 157, 191-200. [CrossRef]

20. Meleán, Y.; Sigalotti, L.D.G. Coalescence of colliding van der Waals liquid drops. Int. J. Heat Mass Transf. 2005, 48, 4041-4061. [CrossRef]

21. Ming, F.; Sun, P.; Zhang, A. Numerical investigation of rising bubbles bursting at a free surface through a multiphase SPH model. Meccanica 2017, 52, 2665-2684. [CrossRef]

22. Gnanasekaran, B.; Liu, G.R.; Fu, Y.; Wang, G.; Niu, W.; Lin, T. A Smoothed Particle Hydrodynamics (SPH) procedure for simulating cold spray process-A study using particles. Surf. Coat. Technol. 2019, 377, 124812. [CrossRef]

23. Sigalotti, L.D.G.; López, H. Adaptive kernel estimation and SPH tensile instability. Comput. Math. Appl. 2008, 55, 23-50. [CrossRef]

24. Milgram, J.H. Active water-wave absorbers. J. Fluid Mech. 1970, 42, 845-859. [CrossRef]

25. Schäffer, H.A.; Klopman, G. Review of Multidirectional Active Wave Absorption Methods. J. Waterw. Port C ASCE 2000, 126, 88-97. [CrossRef]

26. Ouellet, Y.; Datta, I. A survey of wave absorbers. J. Hydraul. Res. 1986, 24, 265-280. [CrossRef]

27. Havelock, T. Forced surface-waves on water. Lond. Edinb. Dubl. Phil. Mag. 1929, 8, 569-576. [CrossRef]

28. Ba Thuy, N.; Tanimoto, K.; Tanaka, N.; Harada, K.; Iimura, K. Effect of open gap in coastal forest on tsunami run-up - investigations by experiment and numerical simulation. Ocean. Eng. 2009, 36, 1258-1269. [CrossRef]

29. Briggs, M.J.; Synolakis, C.E.; Harkins, G.S.; Green, D.R. Laboratory experiments of tsunami runup on a circular island. Pure Appl. Geophys. 1995, 144, 569-593. [CrossRef]

30. Dematteis, G.; Grafke, T.; Onorato, M.; Vanden-Eijnden, E. Experimental Evidence of Hydrodynamic Instantons: The Universal Route to Rogue Waves. Phys. Rev. X 2019, 9, 041057. [CrossRef]

31. McAllister, M.L.; Draycott, S.; Adcock, T.A.A.; Taylor, P.H.; van den Bremer, T.S. Laboratory recreation of the Draupner wave and the role of breaking in crossing seas. J. Fluid Mech. 2019, 860, 767-786. [CrossRef]

32. Ursell, F.; Dean, R.G.; Yu, Y.S. Forced small-amplitude water waves: A comparison of theory and experiment. J. Fluid Mech. 1960, 7, 33-52. [CrossRef]

33. Dean, R.G.; Dalrymple, R.A. Water Wave Mechanics for Engineers and Scientists; World Scientific Publishing Company: Singapore, 1991; Volume 2.

34. Ozbulut, M.; Ramezanzadeh, S.; Yildiz, M.; Goren, O. Modelling of wave generation in a numerical tank by SPH method. J. Ocean. Eng. Mar. Energy 2020, 6, 121-136. [CrossRef]

35. Stoker, J.J. Water Waves: The Mathematical Theory with Applications; John Wiley \& Sons: Hoboken, NJ, USA, 2011 ; Volume 36.

36. Lamb, H.L. Hydrodynamics; Cambridge University Press: Cambridge, UK, 1932.

37. Kajiura, K. The leading wave of a tsunami. B. Earthq. Res. I. Tokyo 1963, 41, 535-571.

38. Munk, W. Origin and generation of waves. Coast. Eng. Proc. 1950, 1, 1. [CrossRef] 
39. McCue, L.; Alford, L.; Belknap, W.; Bulian, G.; Delorme, L.; Francescutto, A.; Vakakis, A. An overview of the minisymposium on extreme ship dynamics presented at the 2005 SIAM conference on applications of dynamical systems. Mar. Technol. 2006, 43, 55-61. Available online: https:/ / onepetro.org/journal-paper/SNAME-MTSN-2006-43-1-55 (accessed on 1 March 2021).

40. Jones, D.A.; Belton, D. Smoothed Particle Hydrodynamics: Applications within DSTO; Technical Report; Defence Science Technology Organisation: Australia, 2006. Available online: https://apps.dtic.mil/sti/pdfs/ADA463727.pdf (accessed on 1 March 2021).

41. Guilcher, P.; Ducorzet, G.; Alessandrini, B.; Ferrant, P. Water wave propagation using SPH models. In Proceedings of the 2nd International SPHERIC Workshop, Madrid, Spain, 23-25 May 2007; pp. 119-124.

42. De Padova, D.; Dalrymple, R.; Mossa, M.; Petrillo, A. SPH simulations of regular and irregular waves and their comparison with experimental data. arXiv 2009, arXiv:0911.1872. 